\title{
Oxygen Line Formation in 3D Hydrodynamical Model Atmospheres
}

\author{
Martin Asplund \\ Astronomiska Observatoriet, Box 515, SE-751 20 Uppsala, Sweden
}

\begin{abstract}
The formation of [O I $], \mathrm{O}_{\mathrm{I}}$ and $\mathrm{OH}$ lines in metal-poor stars has been studied by means of $3 \mathrm{D}$ hydrodynamical model atmospheres. For O I detailed 3D non-LTE calculations have been performed. While the influence of 3D model atmospheres is minor for [OI] and $\mathrm{O}_{\mathrm{I}}$ lines, the very low temperatures encountered at low metallicities have a drastic impact on $\mathrm{OH}$. As a result, the derived $\mathrm{O}$ abundances are found to be systematically overestimated in $1 \mathrm{D}$ analyses, casting doubts on the recent claims for a monotonic increase in $[\mathrm{O} / \mathrm{Fe}]$ towards lower metallicities. ${ }^{1}$
\end{abstract}

\section{Introduction}

Besides being relatively common, oxygen has attracted a great deal of attention due to its special role in Galactic and stellar evolution. The ratio $[\mathrm{O} / \mathrm{Fe}]$ at different cosmic epochs gives important insight to the formation and evolution of the Galaxy, as well as constraining the physics of supernovae. Oxygen can also significantly influence stellar evolution and is crucial for the production of the light elements $\mathrm{Li}, \mathrm{Be}$ and $\mathrm{B}$ through cosmic ray spallation. Following the first indication of an oxygen over-abundance relative to iron in metal-poor stars, a great number of studies have been devoted to quantifying this enhancement but with little concordance. The forbidden [O I] line at $630.0 \mathrm{~nm}$ in metal-poor giants suggests a nearly flat plateau at $[\mathrm{O} / \mathrm{Fe}] \sim 0.5$ for $[\mathrm{Fe} / \mathrm{H}] \lesssim-1.0$ while the $\mathrm{O}$ I triplet at $777 \mathrm{~nm}$ in dwarfs tends to imply systematically higher values, often with a monotonic increase towards lower metallicities. Recently, Israelian et al. (1998) and Boesgaard et al. (1999) have analysed the UV OH lines down to $[\mathrm{Fe} / \mathrm{H}] \simeq-3.3$ and have found a linear trend in $[\mathrm{O} / \mathrm{Fe}]$ vs $[\mathrm{Fe} / \mathrm{H}]$ with a slope of about -0.4 , in stark conflict with the $[\mathrm{O} \mathrm{I}]$ results. All oxygen criteria have their pros and cons, which influence the conclusions. Of particular interest is the possible systematic errors introduced by the inherent assumptions behind the 1D model atmospheres employed in the analyses of the various $O$ diagnostics. Significant progress in this respect has recently been accomplished with the construction of 3D stellar convection simulations (e.g. Stein \& Nordlund 1998; Asplund et al. 1999, 2000a). The aim of the present investigation is to study the impact of such improved models on oxygen line formation.

\footnotetext{
${ }^{1}$ The full version of the present talk with additional figures can be found at http://www.astro.uu.se/ martin/talks/Manchester00
} 

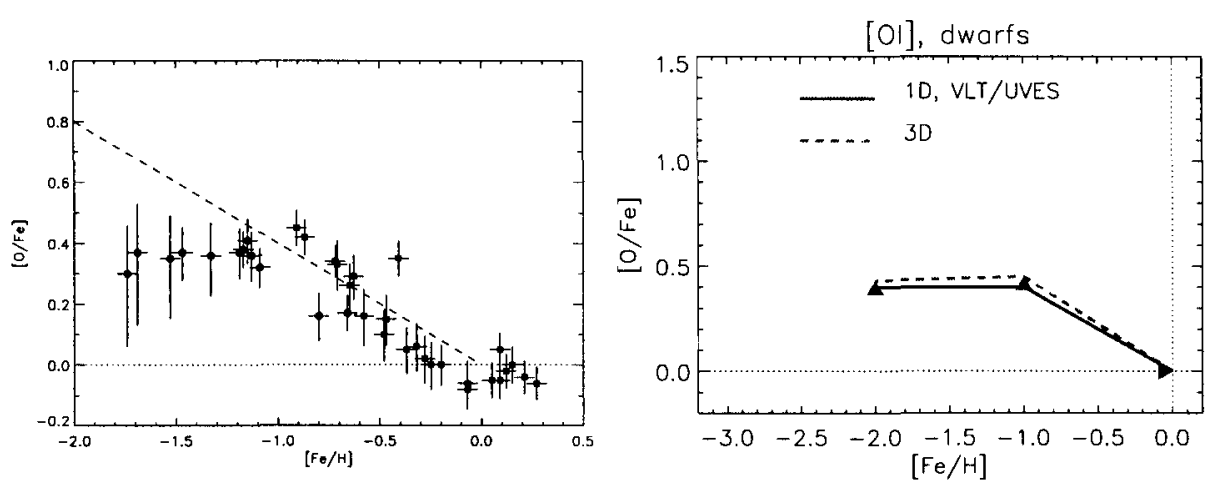

Figure 1. Left panel: $[\mathrm{O} / \mathrm{Fe}]$ ratios determined from $[\mathrm{OI}]$ and Fe II lines from very high $S / N$ VLT spectra (Nissen et al., in preparation). Right panel: The (minor) influence of 3D models on the [O I] line.

\section{3D hydrodynamical model atmospheres and line formation}

Realistic ab-initio 3D, time-dependent simulations of stellar surface convection form the foundation for the present study. The same incompressible radiative hydrodynamical code which previously has been applied successfully to studies of solar and stellar granulation (e.g. Stein \& Nordlund 1998; Asplund et al. 1999, 2000a) has here been used to construct sequences of 3D model atmospheres. Special care has been taken to include the most appropriate input physics in terms of equation-of-state, opacities and line-blanketing. Further details on the numerical procedures may be found in the above-mentioned references.

The 3D model atmospheres differ from classical 1D models both in their average photospheric structures and through the presence of temperature inhomogeneities and convective flows, which all influence the spectral line formation (e.g. Asplund et al. 2000a,b). In particular for metal-poor stars, the 3D simulations differ radically from $1 \mathrm{D}$ model atmospheres (Asplund et al. 1999). Due to the dominance of adiabatic cooling over radiative heating in the optically thin layers, very low temperatures are encountered in the metal-poor simulations compared with the $1 \mathrm{D}$ radiative equilibrium expectations. As a result, the 3D models have a profound impact on the line formation of in particular low-excitation lines of minority species and molecular lines, such as $\mathrm{Li}$ I and $\mathrm{OH}$.

\section{Impact on $\mathbf{O}$ abundances in metal-poor stars}

Since essentially all of $\mathrm{O}$ is in the ground state of $\mathrm{O} I$ and the $[\mathrm{O} I]$ line formation is very well described by LTE, the influence of $3 \mathrm{D}$ models on the $[\mathrm{OI}]$ line is minor. As a consequence, the $[\mathrm{O} / \mathrm{Fe}]$ plateau appears robust, provided high $S / N$ spectra and Fe II lines are employed (Fig. 1 and Nissen et al., in preparation).

Kiselman (these proceedings) has given convincing arguments that the $\mathrm{OI}$ triplet suffers from departures from LTE. The O I line formation has therefore been investigated through detailed 3D non-LTE calculations for the Sun and HD $140283([\mathrm{Fe} / \mathrm{H}]=-2.5)$ using MULTI3D (Botnen 1997) and an extensive 23-level $\mathrm{O}$ model atom. Very similar non-LTE abundance corrections to the 1D non-LTE results are found, typically 0.2 dex with only a minor metallicity 

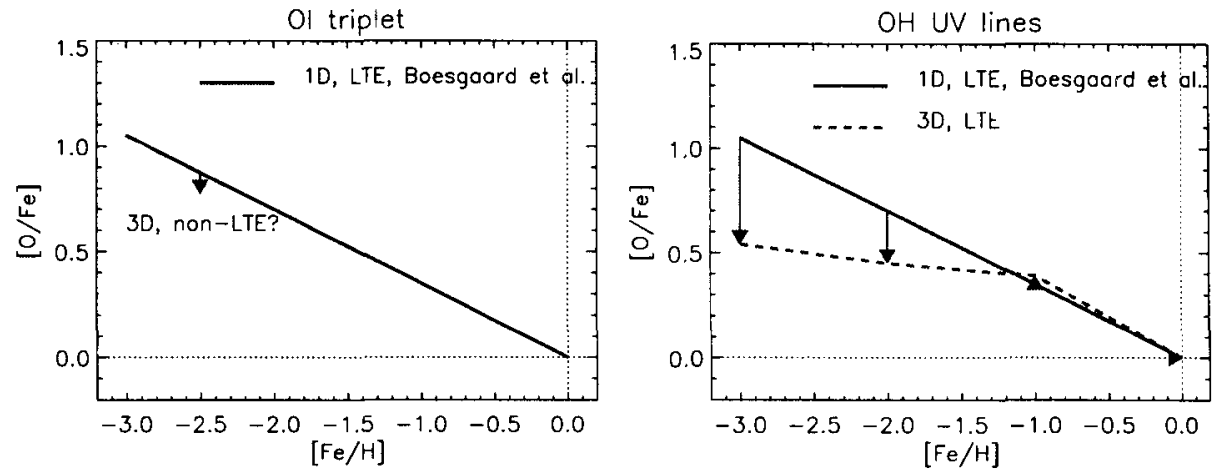

Figure 2. The influence of 3D models on the $\mathrm{O}$ I triplet (Left panel) and the UV OH lines (Right panel).

dependence. Thus, 3D models will unlikely provide a means to bring down the $[\mathrm{O} / \mathrm{Fe}]$ trend to plateau-like values (Fig. 2).

The low atmospheric temperatures in the metal-poor 3D models become very apparent in the $\mathrm{OH}$ line strengths. Since molecular formation is extremely temperature sensitive $\left(N_{\mathrm{OH}} \propto T^{-12 \ldots-14}\right), 1 \mathrm{D}$ model atmospheres are bound to severely underestimate the $\mathrm{OH}$ line strengths, and consequently overestimate the $\mathrm{O}$ abundances at low metallicities (Fig. 2). The here presented 3D LTE OH analysis therefore casts doubts on recent claims of large $\mathrm{O}$ over-abundances in metal-poor stars. The $\mathrm{OH}$ line formation is studied further in Asplund \& García Pérez (2000).

\section{Conclusions}

The new generation of 3D hydrodynamical model atmospheres has a dramatic impact on in particular the $\mathrm{OH}$ line formation in metal-poor stars. Caution must, however, be exercised in interpreting these 3D findings in terms of Galactic cosmic evolution until detailed 3D non-LTE calculations for the $\mathrm{OH}$ molecular formation and line formation have been performed.

Acknowledgments. The author greatly appreciates very stimulating collaborations with among others M. Carlsson, A.E. García Pérez, D. Kiselman, D.L. Lambert, P.E. Nissen, $\AA$. Nordlund and F. Primas.

\section{References}

Asplund M., et al., 1999, A\&A, 346, L17

Asplund M., et al., 2000a, A\&A, 359, 729

Asplund M., et al., 2000b, A\&A, 359, 743

Asplund M., García Pérez A.E., 2000, submitted to A\&A

Boesgaard A.M., et al., 1999, AJ, 117, 492

Botnen A.V., 1997, Cand. Sci. thesis, University of Oslo

Israelian G., et al., 1998, ApJ, 507, 805

Stein R.F., Nordlund $\AA ., ~ 1998$, ApJ, 499, 914 